\title{
Sentinel-Lymph-Node-Biopsie beim malignen Melanom
}

\section{H. Konrad \\ U. Wollina}

\section{Sentinel Lymph Node Biopsy in Malignant Melanoma}

\section{Zusammenfassung}

Die Methode der Schildwächter-Lymphknoten-Biopsie (Sentinel lymph node biopsy, SLNB) ist ein Instrument im Rahmen des Melanom-Stagings, welches bei Tumoren mit einer Dicke $>1 \mathrm{~mm}$ etabliert ist. Das Verfahren ist als minimal invasiver Eingriff weniger risikobelastet als die elektive Lymphknotendissektion. Dennoch sind Komplikationen und unerwünschte Nebenwirkungen des Verfahrens kritisch zu beachten. Bei Patienten mit einem primären Melanom ohne Hinweise auf Metastasierung erlaubt die SLNB eine exakteres Staging, welches letztlich in die weiteren Therapieentscheidungen einfließt. Andererseits sind die Daten zur Prognoseverbesserung bisher noch nicht ausreichend für eine abschließende Beurteilung.
Abstract

The technique of sentinel lymph node biopsy (SLNB) is a tool for melanoma staging that has been established for tumours thicker than $1 \mathrm{~mm}$. The procedure is minimal invasive with reduced risk compared with elective lymph node dissection. Nevertheless, contraindications and adverse effects have to be considered. In patients with a primary melanoma without evidence of spreading SLNB provides a more precise staging that also has an influence on further therapeutic options. On the other hand, data on prognosis improvement by SLNB are yet not sufficient for final confirmation.

\section{Einführung}

Bei primär kutanem Melanom ist die komplette chirurgische Exzision des Tumors mit Sicherheitsabstand die Methode der Wahl. Um die Tumorausbreitung festzustellen werden - falls möglich - noch vor dem operativen Eingriff die Staginguntersuchungen durchgeführt, vor allem die sonographische Untersuchung regionärer Lymphknoten. Der regionäre Lymphknotenstatus ist neben der Tumordicke einer der wichtigsten prognostischen Faktoren, da die regionären Lymphknoten mit Abstand am häufigsten Sitz der Erstmetastasen sind [14].

Ein Teil der Patienten, die klinisch einem N0-Stadium entsprechen, weisen schon Mikrometastasen in den regionären Lymph- knoten auf. Deshalb wurde über einen längeren Zeitraum die prophylaktische Ausräumung der dem Tumor zugehörigen Lymphknotenregion unter der Hypothese durchgeführt, hierdurch möglicherweise bei geringer Tumorlast eine weitere Streuung zu verhindern (vor allem bei Melanomen mit einer größeren Tumordicke). Die Ergebnisse der elektiven Lymphknotendissektion gaben über Jahrzehnte Anlass zu Diskussionen. Von der elektiven Lymphknotendissektion profitierten nur bestimmte Patientengruppen [3].

Von Morton et al. (1992) wurde die Methode der SentinelLymph-Node-Biopsie (SLNB) für das maligne Melanom eingeführt. Die Grundlage dieses Verfahrens basiert auf der Annahme, dass es für jeden Punkt der Haut spezifische kutane Lymphab-

Hautklinik (Chefarzt: Prof. Dr. med. U. Wollina) am Krankenhaus Dresden-Friedrichstadt,

Akademisches Lehrkrankenhaus der TU Dresden, Dresden

Korrespondenzadresse

Prof. Dr. med. U. Wollina · Hautklinik am Krankenhaus Dresden-Friedrichstadt, Akademisches Lehrkrankenhaus der TU Dresden, Dresden · Friedrichstraße 41 · 01067 Dresden ·E-mail: wollina-uw@khdf.de

Bibliografie

Akt Dermatol 2005; 31: 148-150 @ Georg Thieme Verlag KG Stuttgart · New York

DOI $10.1055 / \mathrm{s}-2005-861258 \cdot$ ISSN 0340-2541 
flussbahnen gibt, die zu einem, selten zu mehreren Lymphknoten in der regionären Filterstation führen. Als Sentinel-(Schildwächter)-Lymphknoten (SLN) wird der erste tumordrainierte Lymphknoten bezeichnet. Er gilt als Indikator für die gesamte Lymphknotenregion. Nach herrschender Ansicht werden die ersten Tumorzellen zu Beginn einer lymphogenen Metastasierung im SLN gefunden [10].

Ziel der SLNB ist die Früherkennung von Mikrometastasen eines Melanoms bei noch fehlender Nachweisbarkeit mittels konventioneller Methoden. Die Technik basiert auf der gezielten, minimal invasiven Entnahme des lymphabflussszintigraphisch identifizierten und radioaktiv markierten ersten Filterlymphknotens des Melanoms zur anschließenden histopathologischen Begutachtung. Im Falle eines Mikrometastasennachweises im SLN besteht nach derzeitigem Kenntnisstand die Indikation zur nachfolgenden radikalen Lymphknotendissektion. Bei fehlendem Nachweis kann auch bei Melanomen mit einer großen Tumordicke auf diesen komplikationsbehafteten Eingriff verzichtet werden. Dies ist ein wesentlicher Fortschritt zur früher prophylaktisch durchgeführten Lymphknotendissektion [9].

\section{Indikation zur SLN-Biopsie}

Die SLNB ist ein Staging-Instrument. In den Leitlinien der Deutschen Krebsgesellschaft (2002) wird die SLNB vor allem zur Klassifizierung von Patienten in klinischen Studien erwähnt [2]. In der Praxis hat sich die Methode mittlerweile auch für das Staging außerhalb von Studien durchgesetzt und hat in der Neufassung der Stadieneinteilung des malignen Melanoms durch das American Joint Comittee on Cancer seinen Niederschlag gefunden [4].

Aktuell wird der Eingriff bei Melanomen mit einer Tumordicke $>1 \mathrm{~mm}$ und $<4 \mathrm{~mm}$ sowie fehlendem klinischem Nachweis von Metastasen vorgenommen. Melanome mit einer Tumordicke $<0,75 \mathrm{~mm}$ haben nur selten regionale Lymphknotenmetastasen, hingegen steigt bei Melanomen mit einer Tumordicke größer als $4 \mathrm{~mm}$ das Risiko von Fernmetastasen auf über 70\% [9].

In einer Studie an 146 Patienten mit Melanomen < $1 \mathrm{~mm}$ wurden in $4 \%$ mittels SNLB Metastasen nachgewiesen [15]. Prädiktoren für die Erkennung dieser kleinen Risikogruppe fehlen bisher. Somit bleibt die Feststellung gültig, dass eine SNLB bei dünnen Melanomen keine Routinemethode darstellt.

\section{Methode der Sentinel-Lymph-Node-Biopsie}

In der Umgebung des Primärtumors oder der Narbe nach Exzision wird ein radioaktiver Tracer (zumeist 99m-Technetium-Nanokolloid) intradermal injiziert und durch dynamische Lymphabflussszintigraphie mit der Gamma-Kamera der regionale Lymphabstrom dargestellt $[1,10]$. Neben den drainierenden Lymphbahnen kommt in der regionalen Lymphknotenstation ein Punctum maximum mit Anreicherung des radioaktiven Tracers zur Darstellung (Abb.1). Diese maximale Anreicherung entspricht dem SLN. Die Lokalisation des SLN wird auf der Haut markiert. Vor Beginn des operativen Eingriffes - der auch in Tumes-

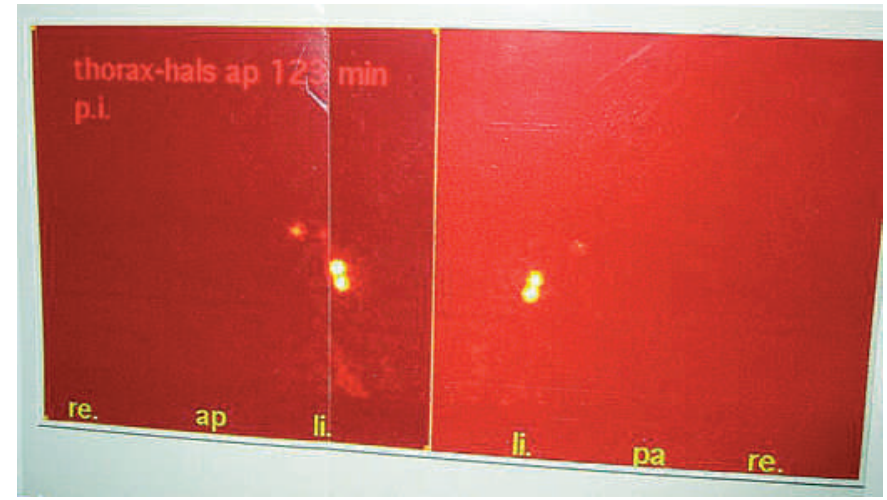

Abb. 1 Lokalisation des Sentinel-Lymphknotens mittels Lymphabflussszintigraphie.

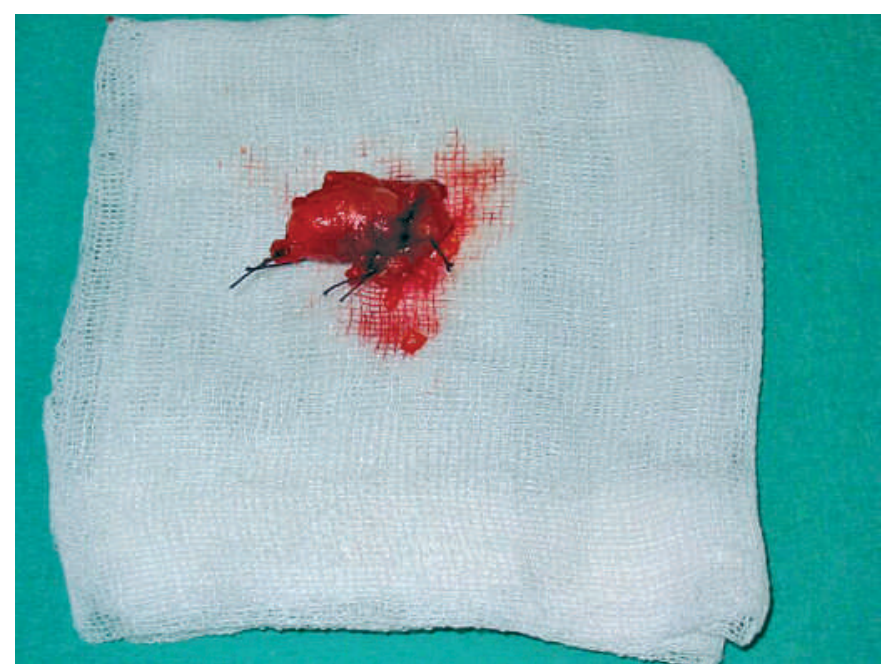

Abb. 2 Op-Präparat eines Sentinel-Lymphknotens.

zenzanalgesie durchgeführt werden kann - wird im Bereich des markierten Areals mit einer für 99m-Tc kollimierten Sonde die Lokalisationsangabe überprüft und über eine kleine Inzision unter Führung der Gamma-Sonde der speichernde Lymphknoten aufgesucht und exstirpiert (Abb.2). Der Eingriff ist beendet wenn sich in der Region keine weiteren nennenswerten radioaktiven Signale mehr nachweisen lassen. Die Strahlenbelastung für den Operateur ist sehr niedrig und liegt bei ca. 0,005 mSv (Millisievert) pro Patient, die Belastung der Op-Schwester etwa ein Zehntel davon. Der SLN wird häufig zusätzlich angefärbt. Dafür wird ca. 10 min vor Op-Beginn periläsional Patentblau-V oder Isosulfanblau injiziert [10] (Abb. 3).

Für nicht voroperierte Patienten verspricht diese Methode eine hohe Sensitivität. Bei Patienten bei welchen bei Entfernung des Primärtumors ausgedehnte Verschiebeplastiken oder freie Hauttransplantationen durchgeführt wurden, kann das Verfahren nicht angewendet werden, da die lokalen kutanen Lymphabstrombahnen zerstört sind. Spezielle Probleme ergeben sich bei Tumoren im Kopf-Hals-Bereich sowie bei Tumoren im Bereich der regionalen Lymphknotenstationen [1,6].

Die intraoperative Detektionsrate des SLN liegt bei etwa $97 \%$. Bei der histopathologischen Begutachtung konnten bei 14 bis $24 \%$ 


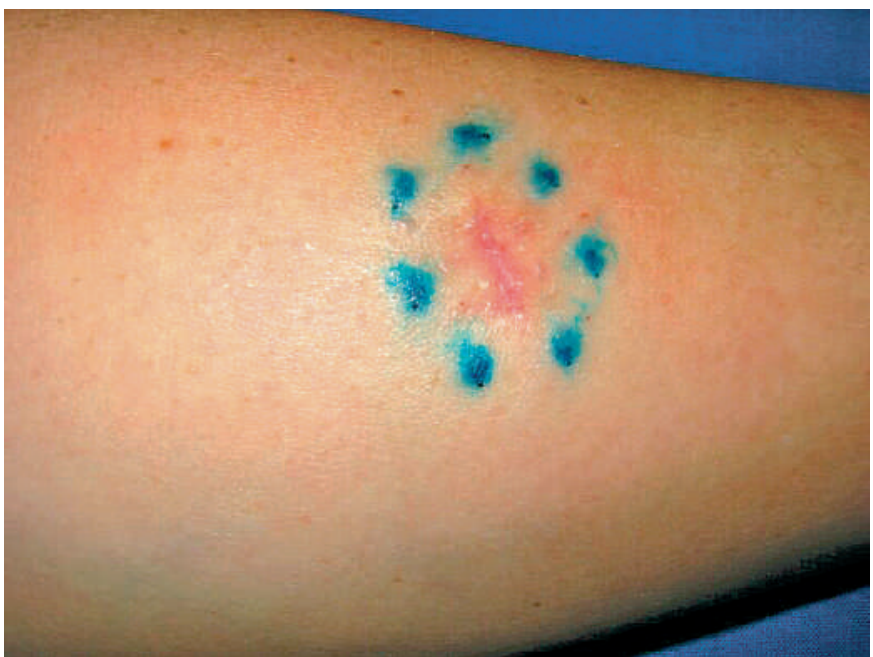

Abb. 3 Zusätzliche peritumorale Patentblau-Injektion zur Lymphknotenmarkierung im OP-Situs (Primäroperation erfolgte ohne Melanomverdacht auswärts).

der so behandelten Patienten Mikrometastasen nachgewiesen werden $[1,9]$.

Bei SLN-positiven Patienten wird eine therapeutische regionale Lymphknotendissektion durchgeführt. Die histopathologische Begutachtung dieser Dissektate zeigte in bis zu $12 \%$ einen metastatischen Befall nachgeordneter Lymphknoten $[1,3,9]$.

Patienten, die einen sehr langsamen Lymphabfluss in der Szintigraphie erkennen lassen $(>30 \mathrm{~min})$, zeigten in einer Studie mit 88 Melanompatienten (Tumordicke $>1 \mathrm{~mm}$ ) in keinem einzigen Fall eine Metastasierung [5]. Möglicherweise kann solchen Patienten eine SLNB erspart werden.

\section{Komplikationen}

Obwohl die SLNB ein minimal invasiver Eingriff ist können Komplikationen auftreten. So gibt es einzelne Berichte über anaphylaktische Reaktionen nach der Applikation von Patentblau und ähnlichen Farbstoffen [7]. Wasserberg et al. (2004) fanden in ihrer 8-Jahresbilanz in 20\% Komplikationen wie Hämatome, Serome, Lymphozelen, Wundinfekte und sensorische Störungen (bes. axillär) [16].

\section{Ausblick}

Mit dem minimal-invasiven Eingriff der SLNB kann das Melanom-Staging in Stadium I und II präziser durchgeführt werden, ohne die höheren Risiken der klassischen Lymphknotendissektion eingehen zu müssen [9]. Ein Mikrometastasen-Nachweis im SLN ist ein unabhängiger prognostischer Parameter $[4,13]$. Die SLBN verbessert somit die Entscheidungsgrundlage über mögliche zusätzliche Behandlungen.
Eine eindeutige Indikation für den regelhaften Einsatz der SLNB wäre ein zusätzlicher therapeutischer Nutzen dieses Eingriffes. Hierzu liegen retrospektive Untersuchungen vor. So konnte in einer retrospektiven Vergleichsstudie an 314 Patienten mit SLNB und 623 Patienten mit Lymphknotendissektion wegen des klinischen/sonographischen Metastasenverdachtes gezeigt werden, dass die 3- und 5-Jahres-Überlebensraten für SLNB-Patienten signifikant günstiger ausfielen [8]. In einer retrospektiven Vergleichsuntersuchung an 283 SLNB-Patienten und 3514 Melanom-Patienten im Stadium I und II mit alleiniger Nachsorge ergab sich für die SLN-positiven Fälle kein Überlebensvorteil [12]. Validere Daten sind von prospektiven multizentrischen Studien zu erwarten.

\section{Literatur}

${ }^{1}$ Alex JC, Krag DN. Gamma-probe-guided lymph node localisation in malignant melanoma. Surg Oncol 1993; 2: 303-308

${ }^{2}$ AMWF Leitlinien (2002) Malignes Melanom der Haut. Register-Nummer 032/024. http://www.uni-duesseldorf.de/WWW/AMWF.

${ }^{3}$ Balch CM, Soong SJ, Bartolucci AA. Efficacy of an elective regional lymph node dissection of 1 to $4 \mathrm{~mm}$ thick melanomas for patients 60 years of age and younger. Ann Surg 1996; 224: 255-266

${ }^{4}$ Balch CM, Buzaid AC, Soong SJ. Final version of the American Joint Committee on Cancer Staging System for cutaneous melanoma. J Clin Oncol 2001; 19: 3635 - 3648

${ }^{5}$ Cammilleri S, Jacob T, Rojat-Habib MC, Hesse S, Berthet B, Giorgi R, Bonerandi JJ, Mundler O. High negative predictive value of slow lymphatic drainage on metastatic node spread detection in malignant limb and trunk cutaneous melanoma. Bull Cancer 2004; 91: E225-E228

${ }^{6}$ Glass LF, Messina JL, Cruse W, Wells K, Rapaport D, Miliotes G, Berman C, Reintgen D, Fenske NA. The use of intraoperative radiolymphoscintigraphy for sentinel node biopsy in patients with malignant melanoma. Dermatol Surg 1996; 22: 715-720

${ }^{7}$ Komenaka IK, Bauer VP, Schnabel FR, Horowitz E, Joseph KA, Ditkoff $\mathrm{BA}$, El-Tamer MB. Allergic reactions to isosulfan blue in sentinel lymph node mapping. Breast J 2005; 11: $70-72$

${ }^{8}$ Kretschmer L, Hilgers R, Möhrle M, Balda BR, Breuninger H, Konz B, Kunte C, Marsch WC, Neumann C, Starz H. Patients with lymphatic metastasis of cutaneous malignant melanoma benefit from sentinel lymphonodectomy and early excision of their nodal disease. Eur J Cancer 2004; 40: 212-218

${ }^{9}$ Lee KK, Vetto JT, Mehrany K, Swanson NA. Sentinel lymph node biopsy. Clin Dermatol 2004; 22: 234-239

${ }^{10}$ Morton DL, Wen DR, Wong JH. Technical details of intraoperative lymphatic mapping for early stage melanoma. Arch Surg 1992; 127: $392-$ 399

${ }^{11}$ Möhrle M, Schippert W, Rassner G, Garbe C, Breuninger H. Is sentinel lymph node biopsy of therapeutic relevance for melanoma? Dermatology 2004; 209: 5 - 13

${ }^{12}$ Möhrle M, Schippert W, Rassner G, Garbe C, Breuninger H. Micrometastasis of a sentinel lymph node in cutaneous melanoma is a significant prognostic factor for disease-free survival, distant-free survival, and overall survival. Dermatol Surg 2004; 30: 1319-1328

${ }^{13}$ Morton DL, Cochran AJ. The case for lymphatic mapping and sentinel lymphadenectomy in the management of primary melanoma. $\mathrm{Br} \mathrm{J}$ Dermatol 2004; 151: $308-319$

14 Petro A, Schwartz J, Johnson T. Current melanoma staging. Clin Dermatol 2004; 22: 223-227

${ }^{15}$ Stitzenberg KB, Groben PA, Stern SL, Thomas NE, Hensing TA, Sansbury LB, Ollila DW. Indications for lymphatic mapping and sentinel lymphadenectomy in patients with thin melanoma (Breslow thickness < or $=1.0 \mathrm{~mm}$ ). Ann Surg Oncol 2004; 11: 900-906

${ }^{16}$ Wasserberg N, Tulchinsky H, Schachter J, Feinmesser M, Gutman H. Sentinel-lymph-node biopsy (SLNB) for melanoma is not complication-free. Eur J Surg Oncol 2004; 30: 851 -856 